\title{
PSA/PSMA DNA Plasmid INO-5150
}

National Cancer Institute

\section{Source}

National Cancer Institute. PSA/PSMA DNA Plasmid INO-5150. NCI Thesaurus. Code C123283.

A plasmid DNA vaccine encoding the tumor-associated antigens (TAAs) prostate-specific antigen (PSA) and prostate-specific membrane antigen (PSMA), with potential immunoactivating and antineoplastic activities. Upon intramuscular delivery and electroporation of the PSA/PSMA DNA plasmid INO-5150, both PSA and PSMA are translated in cells which then activate the immune system. This induces cytotoxic Tlymphocyte $(\mathrm{CTL}$ ) responses against tumor cells expressing PSA and PSMA. This may result in both immune-mediated tumor cell death and the inhibition of tumor cell proliferation. PSA and PSMA are overexpressed on a variety of cancer cell types. The DNA encoding the TAAs in INO-5150 is based on both human and other primate antigen gene sequences. As the plasmid genes differ from the human gene sequences encoding these antigens, INO-5150 may overcome immune tolerance to human TAAs. 